\title{
FURTHER RESULTS ON MEROMORPHIC FUNCTIONS THAT SHARE TWO VALUES WITH THEIR DERIVATIVES
}

\author{
CHUNG-CHUN YANG and PING LI
}

(Received 13 January 2003; revised 22 September 2003)

Communicated by P. C. Fenton

\begin{abstract}
In this paper we continue our previous studies and derive all possible expressions for a meromorphic function and its differential polynomials when they share two finite distinct values $a_{1}, a_{2} \mathrm{CM}$ (counting multiplicities) in majority.
\end{abstract}

2000 Mathematics subject classification: primary $30 \mathrm{D} 35$.

Keywords and phrases: meromorphic function, small function, sharing value, differential polynomial.

\section{Introduction}

Let $f$ denote a nonconstant meromorphic function in the complex plane. We shall use the standard notations in Nevanlinna's value distribution theory of meromorphic functions such as the characteristic function $T(r, f)$, the counting function of the poles $N(r, f)$, and the proximity function $m(r, f)$ (see, for example, [3]). By $S(r, f)$ we denote any quantity satisfying $S(r, f)=o(T(r, f))$ as $r \rightarrow \infty$ possibly outside a set of $r$ of finite linear measure. A meromorphic function $a(\not \equiv \infty)$ is called a small function with respect to $f$ provided that $T(r, a)=S(r, f)$.

For a small function $a$ with respect to two meromorphic functions $f$ and $g$, we say that $f$ and $g$ share $a \mathrm{IM}(\mathrm{CM})$ provided that $f-a$ and $g-a$ have the same zeros ignoring (counting) multiplicities. Obviously, two meromorphic functions will have more common properties if they share more values or small functions. In fact, Nevanlinna [7] has proved the famous 5-value theorem which says that two nonconstant meromorphic functions must be equal if they share five values IM. In general, it is difficult to get relationship between two meromorphic functions when

(C) 2005 Australian Mathematical Society $1446-7887 / 05 \$ A 2.00+0.00$ 
they just share four or less values IM. The number of shared values of two meromorphic functions may be reduced if some additional conditions been added. For instance, it was shown by Lee-Yang [8] that if $f$ is entire and shares two finite values CM with $f^{\prime}$, then $f \equiv f^{\prime}$. Since then, the subject of sharing values between meromorphic or entire functions and their derivatives or linear differential polynomials has been studied by many mathematicians, see, for example, [2, 5, 6, 10]. In 1993, Rüssmann [9] proved the following result: Let $f$ be a meromorphic function and

$$
L(f):=f^{(k)}+a_{k-1} f^{(k-1)}+\cdots+a_{0} f
$$

where $k \geq 2$ and the $a_{j}$ 's are polynomials. If $f$ and $L(f)$ share two distinct values in $\mathbb{C}$ counting multiplicities, then $L(f)=f$ up to some exceptional cases which were also given. Unfortunately this result is not published in any journal. Three years later, Bernstein-Chang-Li [1] obtained a similar result for entire functions of several complex variables. As a special case, they proved that any nonconstant entire function $f$ and its linear differential polynomial $L(f)$ (with all coefficients being small meromorphic functions of $f$ ) must be equal if the two functions share two values CM. In [4] the present authors generalized this result and proved the following

THEOREM A. Let $f$ be a nonconstant entire function and

$$
L(f):=c_{-1}+c_{0} f+c_{1} f^{\prime}+\cdots+c_{n} f^{(n)},
$$

where $c_{i}\left(c_{n} \neq 0\right),(i=-1,0,1, \ldots, n)$ are small meromorphic functions of $f$. Let $a_{1}$ and $a_{2}$ be two distinct values in $\mathbb{C}$. If $f$ and $L(f)$ share $a_{1} C M$, and share $a_{2} I M$, then $f=L(f)$ or $f$ and $L(f)$ have the following expressions

$$
f=a_{2}+\left(a_{1}-a_{2}\right)\left(1-e^{\alpha}\right)^{2}, \quad L(f)=2 a_{2}-a_{1}+\left(a_{1}-a_{2}\right) e^{\alpha},
$$

where $\alpha$ is an entire function.

Recently, Wang [10] improved above result as follows:

THEOREM B. Let $f$ be a nonconstant entire function and $L(f)$ the linear differential polynomial defined in (1). If $f$ and $L(f)$ share two complex numbers $a_{1}$ and $a_{2} I M$, and if $\tau\left(a_{1}\right)>(n+2) /(n+3)$, where $n$ is the highest order of the derivative involved in the $L(f)$, then the conclusion in Theorem A still holds, where

$$
\tau\left(a_{1}\right)=\tau\left(a_{1}, f, L(f)\right)= \begin{cases}\liminf _{r \rightarrow \infty} \frac{\bar{N}_{0}\left(r, 1 /\left(f-a_{1}\right)\right)}{\bar{N}\left(r, 1 /\left(f-a_{1}\right)\right)}, & \text { if } \bar{N}\left(r, 1 /\left(f-a_{1}\right)\right) \neq 0 \\ 1, & \text { otherwise }\end{cases}
$$

is the notation introduced by Mues in [5]. Here $\bar{N}_{0}\left(r, 1 /\left(f-a_{1}\right)\right)$ denote the counting function of those $a_{1}$-points of $f$ and $L(f)$ of the same multiplicities but counted only once. Note that $\tau(c)=1$ for a CM shared value $c$. 
It was shown in [4] that the result in Theorem $B$ is not true if we remove the condition $\tau\left(a_{1}\right)>(n+2) /(n+3)$. It is conjectured, [10], that this number can be replaced by $1 / 2$. When $f$ and $L(f)$ share two values $\mathrm{IM}$, it has been a challenging problem to find some more precise relationships between $f$ and $L(f)$. In the present paper, we prove that the number $(n+2) /(n+3)$ in Theorem B can be replaced by $2 / 3$. As an application, we give all the forms of entire functions $f$ if $f$ share two small functions IM with $c_{-1}+c_{0} f+c_{1} f^{\prime}$, where $c_{-1}, c_{0}$, and $c_{1}$ are small functions of $f$.

Before stating our results, we recall the definitions of sharing small functions in the sense of $\mathrm{IM}^{*}$ or $\mathrm{CM}^{*}$, which is a generalization of the definition in the sense of IM or CM. Let $f$ and $g$ be two nonconstant meromorphic functions, and let $a$ be a small function with respect to $f$ and $g$. Denote by $\bar{N}(r, f=a=g)$ the reduced counting function of the common $a$-points of $f$ and $g$ ignoring the multiplicities, and $\bar{N}_{E}(r, f=a=g)$ the reduced counting function of the common $a$-points of $f$ and $g$ with the same multiplicities.

DEFINITION 1. The small function $a$ is said to be shared by $f$ and $g$ in the the sense of $\mathrm{IM}^{*}$, if

$$
\bar{N}\left(r, \frac{1}{f-a}\right)-\bar{N}(r, f=a=g)=S(r, f),
$$

and

$$
\bar{N}\left(r, \frac{1}{g-a}\right)-\bar{N}(r, f=a=g)=S(r, g) .
$$

Similarly, $a$ is said to be shared by $f$ and $g$ in the sense of $\mathrm{CM}^{*}$, if

$$
\bar{N}\left(r, \frac{1}{f-a}\right)-\bar{N}_{E}(r, f=a=g)=S(r, f),
$$

and

$$
\bar{N}\left(r, \frac{1}{g-a}\right)-\bar{N}_{E}(r, f=a=g)=S(r, g) .
$$

Using [4, Theorem 2] we can easily see that Theorem A remains to be valid when $f$ is a nonconstant meromorphic function satisfying $\bar{N}(r, f)=S(r, f)$, and $f, L(f)$ share a small function $a_{1} \mathrm{CM}^{*}$, and share another small function $a_{2} \mathrm{IM}^{*}$. In fact, Theorem 2 in [4] can be extended further as follows:

THEOREM C. Suppose that $f$ is a nonconstant meromorphic function satisfying $\bar{N}(r, f)=S(r, f)$, and $L(f)$ is the linear differential polynomial defined in (1). Let $a_{1}$ and $a_{2}$ be two distinct small functions of $f$. If $f$ and $L(f)$ share $a_{1} C M^{*}$ and share $a_{2} I M^{*}$, then $f=L(f)$ or $f$ and $L(f)$ have the following expressions

$$
f=a_{2}+\left(a_{1}-a_{2}\right)(1-h)^{2}, \quad L(f)=2 a_{2}-a_{1}+\left(a_{1}-a_{2}\right) h,
$$


where $h$ is a meromorphic function satisfying $\bar{N}(r, h)+\bar{N}(r, 1 / h)=S(r, f)$.

REMARK. Here we would like to point out that the function $e^{\alpha}$ in [4, Theorem 2] should be replaced by $h$ as in Theorem C.

In this paper, by further counting the zeros and poles of the auxiliary functions and using some of our earlier results (see [4]), we are able to improve Theorem $\mathrm{C}$ by proving the following main result:

THEOREM 1.1. Suppose that $f$ is a nonconstant meromorphic function satisfying $\bar{N}(r, f)=S(r, f)$, and $g=L(f)$ is the linear differential polynomial defined in (1). Let $a_{1}$ and $a_{2}$ be two distinct complex numbers. If $\max \left\{\tau\left(a_{1}\right), \tau\left(a_{2}\right)\right\}>1 / 2$, then $f$ and $g$ assume one of the following cases:

(a) $f=g$.

(b) $f=a_{2}+\left(a_{1}-a_{2}\right)(1-h)^{2}$ and $g=2 a_{2}-a_{1}+\left(a_{1}-a_{2}\right) h$, where $h$ is $a$ meromorphic function satisfying $\bar{N}(r, h)+\bar{N}(r, 1 / h)=S(r, f)$.

(c) $f=a_{1}+\left(a_{2}-a_{1}\right)(1-h)^{2}$ and $g=2 a_{1}-a_{2}+\left(a_{2}-a_{1}\right) h$, where $h$ is $a$ meromorphic function satisfying $\bar{N}(r, h)+\bar{N}(r, 1 / h)=S(r, f)$.

(d) There exists an integer $k \geq 3$ such that $k \alpha=\varphi$, where

$$
\begin{aligned}
\alpha & =\frac{f^{\prime}-g^{\prime}}{f-g}-\frac{g^{\prime}}{g-a_{1}}-\frac{g^{\prime}}{g-a_{2}}, \\
\varphi & =\frac{f^{\prime}(f-g)}{\left(f-a_{1}\right)\left(f-a_{2}\right)} .
\end{aligned}
$$

If, furthermore, $\max \left\{\tau\left(a_{1}\right), \tau\left(a_{2}\right)\right\}>2 / 3$, then one of the first three cases above must hold.

When the linear differential polynomial $g$ in Theorem 1.1 is restricted to involve only the first derivative of $f$, we have the following result:

THEOREM 1.2. Suppose that $f$ is a nonconstant meromorphic function satisfying $\bar{N}(r, f)=S(r, f)$, and $g=c_{-1}+c_{0} f+c_{1} f^{\prime}$, where $c_{-1}, c_{0}$ and $c_{1}$ are small meromorphic functions of $f$. Let $a_{1}$ and $a_{2}$ be two distinct small functions of $f$. If $f$ and $g$ share $a_{1}$ and $a_{2} I M^{*}$, then one of the following cases holds

(a) $f=g$.

(b) $f=a_{2}+\left(a_{1}-a_{2}\right)(1-h)^{2}, \quad g=2 a_{2}-a_{1}+\left(a_{1}-a_{2}\right) h$;

(c) $f=a_{1}+\left(a_{2}-a_{1}\right)(1-h)^{2}, \quad g=2 a_{1}-a_{2}+\left(a_{2}-a_{1}\right) h$;

(d) $f=\left(a_{1}+a_{2}\right) / 2+\left(a_{2}-a_{1}\right)(h+1 / h) / 4, \quad g=\left(a_{1}+a_{2}\right) / 2+\left(a_{2}-a_{1}\right) h / 2$, where $h$ is a meromorphic function satisfying $\bar{N}(r, h)+\bar{N}(r, 1 / h)=S(r, f)$. 
COROLLARY 1.3. If $c_{-1}, c_{0}$ and $c_{1}$ are constants and $c_{0} \neq 1, c_{1} \neq 0$, then for any distinct rational functions $a_{1}(z)$ and $a_{2}(z)$, the equation

$$
\left(c_{1} f^{\prime}+c_{0} f+c_{-1}\right)^{2}-2 f\left(c_{1} f^{\prime}+c_{0} f+c_{-1}\right)+\left(a_{1}+a_{2}\right) f-a_{1} a_{2}=0
$$

has no transcendental meromorphic solution.

\section{Lemmas}

Let $f$ be a meromorphic function, and $a$ be a small function of $f$. In the following, $\bar{N}_{(k}(r, 1 /(f-a))$ is defined to be the counting function of all zeros of $f(z)-a(z)$ with multiplicities greater than or equal to $k$, and any such zero is counted once only; $\bar{N}_{[k]}(r, 1 /(f-a))$ is defined similarly, but it counts the zeros of $f(z)-a(z)$ with multiplicities $k$.

LEMMA 2.1 ([4]). Suppose that $f$ is a nonconstant meromorphic function satisfying $\bar{N}(r, f)=S(r, f)$, and $g=L(f)$ is the linear differential polynomial defined in (1). Furthermore, let $a_{1}$ and $a_{2}$ be two distinct small functions of $f$. If $f$ and $g$ share $a_{1}$ and $a_{2} I M^{*}$, and if $\neq \mathrm{g}$, then

$$
\begin{aligned}
& T(r, f)=\bar{N}\left(r, \frac{1}{f-a_{1}}\right)+\bar{N}\left(r, \frac{1}{f-a_{2}}\right)+S(r, f), \\
& T(r, f) \leq 2 T(r, g)+S(r, f) .
\end{aligned}
$$

LEMMA 2.2 ([4]). Suppose that $f$ is a nonconstant meromorphic function satisfying $\bar{N}(r, f)=S(r, f)$, and $g=L(f)$ is the linear differential polynomial defined in (1). Furthermore, let $a_{1}$ and $a_{2}$ be two distinct complex numbers. If $f$ and $g$ share $a_{1}$ and $a_{2} I M^{*}$, and iff $\neq g$, then $\sum_{j=0}^{n} c_{j} \varphi_{j}=0$, where $\varphi_{j}$ is defined by the recurrence formula

$$
\varphi_{j+1}=\varphi_{j}^{\prime}+\varphi \varphi_{j}, \quad \varphi_{0}=1, \quad j=0,1, \ldots, n-1,
$$

and $\varphi$ is the function defined in (3).

LEMMA 2.3. Suppose that $f$ is a nonconstant meromorphic function satisfying $\bar{N}(r, f)=S(r, f)$, and $g=L(f)$ is the linear differential polynomial defined in (1). Furthermore, let $a_{1}$ and $a_{2}$ be two distinct small functions of $f$. If $f$ and $g$ share $a_{1}$ and $a_{2} I M^{*}$, and if $T(r, f)=T(r, g)+S(r, f)$, then $f=g$.

PROOF. With loss of generality, we assume that both $a_{1}$ and $b_{2}$ are complex numbers, otherwise, do the following transformation

$$
F=\left(f-a_{1}\right) /\left(a_{2}-a_{1}\right), \quad G=\left(g-a_{1}\right) /\left(a_{2}-a_{1}\right) .
$$


Since $f$ and $g$ share $a_{1}$ and $a_{2} \mathrm{IM}^{*}$, we see that $F$ and $G$ share $0,1 \mathrm{IM}^{*}$, and $G$ is a differential polynomial of $F$ with all coefficients being small functions of $F$.

Let

$$
\psi=\frac{g^{\prime}(f-g)}{\left(g-a_{1}\right)\left(g-a_{2}\right)} .
$$

Since $T(r, f)=T(r, g)+S(r, f)$, by the proof of Lemma 2.2 (see [4, page 353]), we can get $T(r, \psi)=S(r, f)$, and $f=g$.

LEMMA 2.4. Suppose that $f$ is a nonconstant meromorphic function satisfying $\bar{N}(r, f)=S(r, f)$, and $g=L(f)$ is the linear differential polynomial defined in (1). Furthermore, let $a_{1}$ and $a_{2}$ be two distinct small functions of $f$. If $f$ and $g$ share $a_{1}$ and $a_{2} I M^{*}$, and if $\neq \mathrm{g}$, then

$$
\bar{N}_{12}\left(r, \frac{1}{g-a_{1}}\right)+\bar{N}_{(2}\left(r, \frac{1}{g-a_{2}}\right)=S(r, f) .
$$

PROOF. Without loss of generality, we assume that both $a_{1}$ and $a_{2}$ are complex numbers. Let $\varphi$ be the function defined in (3). Since $f$ and $g$ share $a_{1}, a_{2} \mathrm{IM}^{*}$, and $f \neq g$, it is easily seen that $\varphi \neq 0$, and $T(r, \varphi)=S(r, f)$. Rewrite (3) as

$$
f^{\prime}=\varphi\left(f-a_{1}\right)+w_{1}\left(g-a_{2}\right),
$$

where $w_{1}=f^{\prime} /\left(f-a_{2}\right)$. Taking derivative and replacing $f^{\prime}$ by the right-hand side of $(8)$, we get

$$
f^{\prime \prime}=\varphi_{2}\left(f-a_{1}\right)+w_{2}\left(g-a_{2}\right),
$$

where $\varphi_{2}=\varphi^{\prime}+\varphi^{2}$, and

$$
w_{2}=w_{1}^{\prime}+\varphi w_{1}+w_{1} g^{\prime} /\left(g-a_{2}\right) .
$$

Similarly, using (8), we get

$$
f^{(j)}=\varphi_{j}\left(f-a_{1}\right)+w_{j}\left(g-a_{2}\right), \quad j=1,2, \ldots,
$$

where $\varphi_{j}$ and $w_{j}$ are defined by the following recurrence formulae

$$
\begin{aligned}
\varphi_{j+1} & =\varphi_{j}^{\prime}+\varphi \varphi_{j}, \quad \varphi_{0}=1, & j & =0,1, \ldots, \\
w_{j+1} & =w_{j}^{\prime}+w_{1} \varphi_{j}+w_{j} \frac{g^{\prime}}{g-a_{2}}, & j & =1,2, \ldots
\end{aligned}
$$

From (11) and by the definition of $g$, we get

$$
g=c_{-1}+c_{0} a_{1}+\left(\sum_{j=0}^{n} c_{j} \varphi_{j}\right)\left(f-a_{1}\right)+\left(\sum_{j=1}^{n} c_{j} w_{j}\right)\left(g-a_{2}\right)
$$


By Lemma 2.2, we have $\sum_{j=0}^{n} c_{j} \varphi_{j}=0$. Hence

$$
g=c_{-1}+c_{0} a_{1}+\left(\sum_{j=1}^{n} c_{j} w_{j}\right)\left(g-a_{2}\right)
$$

Suppose that $z_{0}$ is an $a_{2}$-point of $g$ of multiplicity $k \geq 2$ as well as a simple $a_{2}$-point of $f$, and $z_{0}$ is not the pole or zero of any $c_{j}$. Then we have

$$
\frac{w_{1}^{\prime}(z)}{w_{1}(z)}+\frac{g^{\prime}(z)}{g(z)-a_{2}}=\frac{k-1}{z-z_{0}}+O(1) .
$$

Equality (15) and (10) imply that $z_{0}$ is a pole of $w_{2}$ of multiplicity 2. By recurrence formula (13), we can see that $z_{0}$ is a pole of $w_{j}$ of multiplicity $j$. Therefore, from (14), we get $k \geq n$. Hence the multiplicities of 'almost all' multiple $a_{2}$-points of $g$ are great than or equal to $n$.

Suppose that $z_{1}$ is an $a_{2}$-point of $g$ of multiplicity $k \geq n+1$. It follows from (14) that $a_{2}=c_{-1}\left(z_{1}\right)+c_{0}\left(z_{1}\right) a_{1}$. If $\bar{N}_{(n+1}\left(r, 1 /\left(g-a_{2}\right)\right) \neq S(r, f)$, then we get $a_{2}=c_{-1}+c_{0} a_{1}$. Therefore, by (14), we get $\sum_{j=1}^{n} c_{j} w_{j}=1$. This is impossible because $z_{1}$ is a pole of $w_{j}$ of multiplicity $j$. Hence $\bar{N}_{(n+1}\left(r, 1 /\left(g-a_{2}\right)\right)=S(r, f)$, and thus

$$
\bar{N}_{(2}\left(r, \frac{1}{g-a_{2}}\right)=\bar{N}_{[n]}\left(r, \frac{1}{g-a_{2}}\right)+S(r, f) .
$$

On the other hand, the $a_{2}$-points of $g$ of multiplicity $n$ are poles of $w_{n+1}\left(g-a_{2}\right)$. By (11), these points must be poles of $f^{(n+1)}-\varphi_{n+1}\left(f-a_{1}\right)$. Note that $f$ is a function satisfying $\bar{N}(r, f)=S(r, f)$. We get

$$
\bar{N}_{[n]}\left(r, \frac{1}{g-a_{2}}\right)=S(r, f)
$$

Hence $\bar{N}_{(2}\left(r, 1 /\left(g-a_{2}\right)\right)=S(r, f)$. Similarly, we have $\bar{N}_{(2}\left(r, 1 /\left(g-a_{1}\right)\right)=S(r, f)$. This completes the proof of Lemma 2.4 .

\section{Proofs of the results}

Proof OF THEOREM 1.1. Let

$$
\beta_{j} \doteq \frac{f^{\prime}}{f-a_{j}}-\frac{g^{\prime}}{g-a_{j}}, \quad j=1,2 .
$$

By the lemma of the logarithmic derivative, we see that $\beta_{j}(j=1,2)$ are meromorphic functions satisfying $m\left(r, \beta_{j}\right)=S(r, f)$. Since $f$ and $g$ share $a_{1}$ and $a_{2} \mathrm{IM}^{*}$, we have

$$
T\left(r, \beta_{j}\right)=\bar{N}\left(r, \frac{1}{f-a_{j}}\right)-\bar{N}\left(r, f=a_{j}=g\right)+S(r, f), \quad j=1,2 .
$$


Suppose that $z_{1}$ is an $a_{1}$-point of $f$ of multiplicity $k$ as well as a simple $a_{1}$-point of $g$. By computation, we get

$$
\varphi\left(z_{1}\right)=k \frac{f^{\prime}\left(z_{1}\right)-g^{\prime}\left(z_{1}\right)}{a_{1}-a_{2}}=k \beta_{2}\left(z_{1}\right) .
$$

If there exists a positive integer $k$ such that $\varphi-k \beta_{2}=0$, then $T\left(r, \beta_{2}\right)=T(r, \varphi / k)=$ $S(r, f)$. Hence $f$ and $g$ share $a_{2} \mathrm{CM}^{*}$. By Theorem C, Case (b) must hold. Similarly, if there exists a positive integer $k$ such that $\varphi-k \beta_{1}=0$, then Case (c) must hold.

In the following, we assume that $\varphi-k \beta_{1} \neq 0$ and $\varphi-k \beta_{2} \neq 0$ for any integer $k$. Then we have

$$
\bar{N}_{[k]}\left(r, \frac{1}{f-a_{1}}\right) \leq \bar{N}\left(r, \frac{1}{\varphi-k \beta_{2}}\right) \leq T\left(r, \beta_{2}\right)+S(r, f), \quad k=1,2, \ldots
$$

Similarly,

$$
\bar{N}_{[k \mid}\left(r, \frac{1}{f-a_{2}}\right) \leq \bar{N}\left(r, \frac{1}{\varphi-k \beta_{1}}\right) \leq T\left(r, \beta_{1}\right)+S(r, f), \quad k=1,2, \ldots
$$

By Lemma 2.4, we have $\bar{N}_{(2}\left(r, 1 /\left(g-a_{j}\right)\right)=S(r, f), j=1,2$. Therefore,

$$
\bar{N}_{[1]}\left(r, \frac{1}{f-a_{j}}\right)=\bar{N}_{E}\left(r, f=a_{j}=g\right)+S(r, f), \quad j=1,2 .
$$

Let $w_{1}=f^{\prime} /\left(f-a_{2}\right)$. We have $T\left(r, w_{1}\right)=\bar{N}\left(r, 1 /\left(f-a_{2}\right)\right)+S(r, f)$. It is obvious that any $a_{1}$-point of $f$ of multiplicity $k$ is a zero of $w_{1}$ of multiplicity $k-1$. Therefore,

$$
\begin{array}{r}
\bar{N}_{|2|}\left(r, \frac{1}{f-a_{1}}\right)+2 \bar{N}_{|3|}\left(r, \frac{1}{f-a_{1}}\right)+3 \bar{N}_{(4}\left(r, \frac{1}{f-a_{1}}\right) \\
\quad \leq N\left(r, \frac{1}{w_{1}}\right) \leq T\left(r, w_{1}\right) \leq \bar{N}\left(r, \frac{1}{f-a_{2}}\right)+S(r, f) .
\end{array}
$$

It follows that

$$
\begin{aligned}
& \frac{1}{3} \bar{N}_{|2|}\left(r, \frac{1}{f-a_{1}}\right)+\frac{2}{3} \bar{N}_{|3|}\left(r, \frac{1}{f-a_{1}}\right)+\bar{N}_{(4}\left(r, \frac{1}{f-a_{1}}\right) \\
& \leq \frac{1}{3} \bar{N}\left(r, \frac{1}{f-a_{2}}\right)+S(r, f) .
\end{aligned}
$$

From (17), (18) and the above inequality, we deduce that

$$
\begin{aligned}
& \bar{N}\left(r, \frac{1}{f-a_{1}}\right)-\bar{N}_{E}\left(r, f=a_{1}=g\right) \\
& \quad=\bar{N}_{|2|}\left(r, \frac{1}{f-a_{1}}\right)+\bar{N}_{|3|}\left(r, \frac{1}{f-a_{1}}\right)+\bar{N}_{44}\left(r, \frac{1}{f-a_{1}}\right)+S(r, f)
\end{aligned}
$$


[9]

$$
\begin{aligned}
= & \frac{2}{3} \bar{N}_{[2]}\left(r, \frac{1}{f-a_{1}}\right)+\frac{1}{3} \bar{N}_{[3]}\left(r, \frac{1}{f-a_{1}}\right)+\frac{1}{3} \bar{N}_{[2]}\left(r, \frac{1}{f-a_{1}}\right) \\
& +\frac{2}{3} \bar{N}_{[3]}\left(r, \frac{1}{f-a_{1}}\right)+\bar{N}_{(4}\left(r, \frac{1}{f-a_{1}}\right)+S(r, f) \\
\leq & {\left[\bar{N}\left(r, \frac{1}{f-a_{2}}\right)-\bar{N}_{E}\left(r, f=a_{2}=g\right)\right]+\frac{1}{3} \bar{N}\left(r, \frac{1}{f-a_{2}}\right)+S(r, f) } \\
= & \frac{4}{3} \bar{N}\left(r, \frac{1}{f-a_{2}}\right)-\bar{N}_{E}\left(r, f=a_{2}=g\right)+S(r, f) .
\end{aligned}
$$

On the other hand, from (17)-(20), we get

$$
\bar{N}_{E}\left(r, f=a_{1}=g\right)+\bar{N}_{E}\left(r, f=a_{2}=g\right) \leq \bar{N}\left(r, \frac{1}{f-a_{j}}\right)+S(r, f)
$$

for $j=1,2$. Hence

$$
\bar{N}_{E}\left(r, f=a_{2}=g\right) \leq \frac{2}{3} \bar{N}\left(r, \frac{1}{f-a_{2}}\right)+S(r, f),
$$

which implies that $\tau\left(a_{2}\right) \leq 2 / 3$. The inequality $\tau\left(a_{1}\right) \leq 2 / 3$ can be obtained similarly. Therefore, the condition $\max \left\{\tau\left(a_{1}\right), \tau\left(a_{2}\right)\right\}>2 / 3$ implies that one of the first three cases in Theorem 1.1 must hold.

Let $\alpha$ be the function defined in (2). From (3), we see that the zeros of $f-g$ must be the zeros of $\varphi$ as long as they are not the $a_{1}$-points or $a_{2}$-points of $f$. Furthermore, the multiple zeros of $f-g$ must be the zeros of $\varphi$. Therefore, 'almost all' of the zeros of $f-g$ are simple. By Lemma 2.4, 'almost all' of the $a_{j}$-points $(j=1,2)$ of $g$ are also simple. Hence $T(r, \alpha)=S(r, f)$. By computation, we see that the equation

$$
k \alpha(z)-\varphi(z)=0
$$

holds for 'almost all' $a_{j}$-points $(j=1,2)$ of $f$ of multiplicity $k \geq 3$. If

$$
\bar{N}_{(3}\left(r, \frac{1}{f-a_{1}}\right)+\bar{N}_{3}\left(r, \frac{1}{f-a_{2}}\right) \neq S(r, f),
$$

then there exists an integer $k \geq 3$ such that $k \alpha-\varphi=0$. Therefore, Case (d) in Theorem 1.1 holds. If

$$
\bar{N}_{(3}\left(r, \frac{1}{f-a_{1}}\right)+\bar{N}_{(3}\left(r, \frac{1}{f-a_{2}}\right)=S(r, f),
$$

then from (21), (20) and (19) we can deduce that

$$
\bar{N}_{E}\left(r, f=a_{1}=g\right) \leq \bar{N}\left(r, \frac{1}{f-a_{2}}\right)-\bar{N}_{E}\left(r, f=a_{2}=g\right)+S(r, f)
$$




$$
\begin{aligned}
& =\bar{N}_{[2]}\left(r, \frac{1}{f-a_{2}}\right)+\bar{N}_{(3}\left(r, \frac{1}{f-a_{2}}\right)+S(r, f) \\
& \leq \bar{N}\left(r, \frac{1}{f-a_{1}}\right)-\bar{N}_{E}\left(r, f=a_{1}=g\right)+S(r, f),
\end{aligned}
$$

which implies $2 \bar{N}_{E}\left(r, f=a_{1}=g\right) \leq \bar{N}\left(r, 1 /\left(f-a_{1}\right)\right)+S(r, f)$. Hence $\tau\left(a_{1}\right) \leq 1 / 2$. Similarly, we can get $\tau\left(a_{2}\right) \leq 1 / 2$. Therefore, the condition $\max \left\{\tau\left(a_{1}\right), \tau\left(a_{2}\right)\right\}>1 / 2$ implies that one of the four cases in Theorem 1.1 must hold. This completes the proof of Theorem 1.1.

Proof of TheOREM 1.2. Suppose that $f \neq g$. Then by Lemma 2.4 'almost all' $a_{j}$-points $(j=1,2)$ of $g$ are simple. If $\bar{N}_{(2}\left(r, 1 /\left(f-a_{1}\right)\right)=S(r, f)$, then $f$ and $g$ share $a_{1} \mathrm{CM}^{*}$. By Theorem A, Case (b) holds. Similarly, Case (c) holds provided that $\bar{N}_{(2}\left(r, 1 /\left(f-a_{2}\right)\right)=S(r, f)$.

In the following, we assume that $\bar{N}_{(2}\left(r, 1 /\left(f-a_{j}\right)\right) \neq S(r, f)$ for $j=1,2$. Note that $a_{j}(z)=c_{-1}(z)+c_{0}(z) a_{j}(z)$ holds for 'almost all' $a_{j}$-points of $f$ and $g$. We have

$$
a_{j}=c_{-1}+c_{0} a_{j}, \quad j=1,2 .
$$

Since $a_{1} \neq a_{2}$, it follows from (22) that $c_{0}=1$ and $c_{-1}=0$. Hence $g=f+c_{1} f^{\prime}$. This and (3) lead to

$$
(f-g)^{2}=-c_{1} \varphi\left(f-a_{1}\right)\left(f-a_{2}\right) .
$$

If $-c_{1} \varphi \neq 1$, then $g(g-2 f)=-c_{1} \varphi\left(f-a_{1}\right)\left(f-a_{2}\right)-f^{2}$ is a polynomial in $f$ of degree 2. Therefore,

$$
T(r, g(g-2 f))=2 T(r, f)+S(r, f) .
$$

Note that $g-2 f$ is linear differential polynomial in $f$, hence $T(r, g-2 f) \leq$ $T(r, f)+S(r, f)$. Thus

$$
T(r, g(g-2 f) \leq T(r, g)+T(r, f)+S(r, f) \leq 2 T(r, f)+S(r, f) .
$$

Hence $T(r, f)=T(r, g)+S(r, f)$. It follows that $f=g$ by Lemma 2.3, which contradicts the assumption.

If $-c_{1} \varphi=1$, then (23) becomes $(f-g)^{2}=\left(f-a_{1}\right)\left(f-a_{2}\right)$, which leads to

$$
f=\frac{g^{2}-a_{1} a_{2}}{2 g-a_{1}-a_{2}} .
$$

Note that $f$ is a function satisfying $\bar{N}(r, f)=S(r, f)$ and $g=L(f)$. Equation (26) implies

$$
\bar{N}(r, h)+\bar{N}(r, 1 / h)=S(r, f)
$$


where $h=\left(2 g-a_{1}-a_{2}\right) /\left(a_{2}-a_{1}\right)$. Obviously, $f$ and $g$ can be expressed as follows

$$
f=\frac{a_{1}+a_{2}}{2}+\frac{a_{2}-a_{1}}{4}\left(h+\frac{1}{h}\right), \quad g=\frac{a_{1}+a_{2}}{2}+\frac{a_{2}-a_{1}}{2} h,
$$

which completes the proof of Theorem 1.2.

PROOF OF COROLLARY 1.3. If $f$ is a transcendental meromorphic solution of (4), then

$$
(f-g)^{2}=\left(f-a_{1}\right)\left(f-a_{2}\right),
$$

where $g=c_{1} f^{\prime}+c_{0} f+c_{-1}$. From (27), we can see that $f$ and $g$ share both $a_{1}$ and $a_{2} \mathrm{IM}^{*}$, and $\bar{N}(r, f)=S(r, f)$. By Theorem 1.2, we get

$$
\begin{aligned}
& f=\frac{a_{1}+a_{2}}{2}+\frac{a_{2}-a_{1}}{4}\left(h+\frac{1}{h}\right), \\
& g=\frac{a_{1}+a_{2}}{2}+\frac{a_{2}-a_{1}}{2} h,
\end{aligned}
$$

where $h$ is a meromorphic function satisfying $\bar{N}(r, h)+\bar{N}(r, 1 / h)=S(r, f)$. Since $g=c_{1} f^{\prime}+c_{0} f+c_{-1}$, it follows from (28) that

$$
\begin{aligned}
g= & c_{-1}+c_{0} \frac{a_{1}+a_{2}}{2}+\left(c_{0} \frac{a_{2}-a_{1}}{4}+c_{1}\left(\frac{a_{2}^{\prime}-a_{1}^{\prime}}{4}+\frac{a_{2}-a_{1}}{4} \frac{h^{\prime}}{h}\right)\right) h \\
& +c_{1} \frac{a_{1}^{\prime}+a_{2}^{\prime}}{2}+\left(c_{0} \frac{a_{2}-a_{1}}{4}+c_{1}\left(\frac{a_{2}^{\prime}-a_{1}^{\prime}}{4}-\frac{a_{2}-a_{1}}{4} \frac{h^{\prime}}{h}\right)\right) \frac{1}{h} .
\end{aligned}
$$

This and (29) lead to

$$
\begin{aligned}
c_{-1}+c_{0} \frac{a_{1}+a_{2}}{2}+c_{1} \frac{a_{1}^{\prime}+a_{2}^{\prime}}{2} & =\frac{a_{1}+a_{2}}{2}, \\
c_{0} \frac{a_{2}-a_{1}}{4}+c_{1}\left(\frac{a_{2}^{\prime}-a_{1}^{\prime}}{4}+\frac{a_{2}-a_{1}}{4} \frac{h^{\prime}}{h}\right) & =\frac{a_{2}-a_{1}}{2}, \\
c_{0} \frac{a_{2}-a_{1}}{4}+c_{1}\left(\frac{a_{2}^{\prime}-a_{1}^{\prime}}{4}-\frac{a_{2}-a_{1}}{4} \frac{h^{\prime}}{h}\right) & =0 .
\end{aligned}
$$

From the last two equations, we get $\left(1-c_{0}\right)\left(a_{2}-a_{1}\right)=c_{1}\left(a_{2}^{\prime}-a_{1}^{\prime}\right)$. Therefore, there exist a nonzero constant $A$ such that $a_{2}(z)-a_{1}(z)=A e^{\left(1-c_{1}\right) z / c}$, which is not any rational function, and contradicts the assumption. This also completes the proof of Corollary 1.3. 


\section{Concluding remarks}

(i) It seems to be an interesting and challenging problem to find the least nonnegative number $d$ such that Theorem 1.1 remains to be valid when $\max \left(\tau\left(a_{1}\right), \tau\left(a_{2}\right)\right)>d$.

(ii) We wonder whether Theorem 1.2 is true if $g$ is replaced by an arbitrary linear differential polynomial.

\section{References}

[1] C. Bernstein, D.-C. Chang and B.-Q. Li, 'On uniqueness of entire functions in $C^{n}$ and their partial differential polynomials', Forum Mathematicum 8 (1996), 379-396.

[2] G. Gundersen, 'Meromorphic functions that share finite values with their derivative', J. Math. Anal. Appl. 75 (1980), $441-446$.

[3] W. Hayman, Meromorphic functions (Clarendon Press, Oxford, 1964).

[4] P. Li and C.-C. Yang, 'Value sharing of an entire function and its derivative', J. Math. Soc. Japan (4) 51 (1999), 781-799.

[5] E. Mues, 'Meromorphic functions sharing four values', Complex Variables 12 (1989), 167-179.

[6] E. Mues and N. Steinmetz, 'Meromorphe Funktionen, die mit ihrer Ableitung Werte teilen', Manuscripta Math. 29 (1979), 195-206.

[7] R. Nevanlinna, 'Einige Eindentigkeitssätze in der Theorie der Meromorphen Funktionen', Acta Math. 48 (1926), 367-391.

[8] L. Rubel and C.-C. Yang, Values shared by an entire function and its derivative, Lecture Notes in Math. 599 (Springer, Berlin, 1977) pp. 101-103.

[9] P. Rüssmann, Über differentialpolynome, die mit ibres erzeugenden Funktion zwei werbe teilen (Ph.D. Thesis, Technischen Universität, Berlin, 1993).

[10] J.-P. Wang, 'Entire functions that share two values IM with their derivatives', Pure Appl. Math. (Chinese) (2) 17 (2001), 142-148.

Department of Mathematics

Hong Kong Univ. of Sci. and Tech.

Kowloon

Hong Kong

P. R. China
Department of Mathematics Univ. of Sci. and Tech. of China

Hefei

Anhui

P. R. China

e-mail: pli@ustc.edu.cn 